\title{
Article \\ Rhizosphere Microbiomes of Potato Cultivated under Bacillus subtilis Treatment Influence the Quality of Potato Tubers
}

\author{
Jian Song ${ }^{\dagger}$, Zhi-Qiang Kong ${ }^{\dagger}$, Dan-Dan Zhang, Jie-Yin Chen $\mathbb{( D}$, Xiao-Feng Dai $\mathbb{1}$ and Ran Li ${ }^{*}$ \\ State Key Laboratory for Biology of Plant Diseases and Insect Pests, Institute of Plant Protection, \\ Chinese Academy of Agricultural Sciences, Beijing 100193, China; songjian_01@126.com (J.S.); \\ kongzhiqiang@caas.cn (Z.-Q.K.); zhangdandan@caas.cn (D.-D.Z.); chenjieyin@caas.cn (J.-Y.C.); \\ daixiaofeng_caas@126.com (X.-F.D.) \\ * Correspondence: liran01@caas.cn \\ † These authors contribute equally to this work.
}

Citation: Song, J.; Kong, Z.-Q.; Zhang, D.-D.; Chen, J.-Y.; Dai, X.-F.; Li, R. Rhizosphere Microbiomes of Potato Cultivated under Bacillus subtilis Treatment Influence the Quality of Potato Tubers. Int. J. Mol. Sci. 2021, 22, 12065. https:// doi.org/10.3390/ijms222112065

Received: 15 September 2021 Accepted: 2 November 2021 Published: 8 November 2021

Publisher's Note: MDPI stays neutral with regard to jurisdictional claims in published maps and institutional affiliations.

Copyright: (c) 2021 by the authors. Licensee MDPI, Basel, Switzerland. This article is an open access article distributed under the terms and conditions of the Creative Commons Attribution (CC BY) license (https:// creativecommons.org/licenses/by/ $4.0 /)$.

\begin{abstract}
Plants serve as a niche for the growth and proliferation of a diversity of microorganisms. Soil microorganisms, which closely interact with plants, are increasingly being recognized as factors important to plant health. In this study, we explored the use of high-throughput DNA sequencing of the fungal ITS and bacterial 16S for characterization of the fungal and bacterial microbiomes following biocontrol treatment (DT) with Bacillus subtilis strain Bv17 relative to treatments without biocontrol (DC) during the potato growth cycle at three time points. A total of 5631 operational taxonomic units (OTUs) were identified from the $16 \mathrm{~S}$ data, and 2236 OTUs were identified from the ITS data. The number of bacterial and fungal OTU in DT was higher than in DC and gradually increased during potato growth. In addition, indices such as Ace, Chao, Shannon, and Simpson were higher in DT than in DC, indicating greater richness and community diversity in soil following the biocontrol treatment. Additionally, the potato tuber yields improved without a measurable change in the bacterial communities following the B. subtilis strain Bv17 treatment. These results suggest that soil microbial communities in the rhizosphere are differentially affected by the biocontrol treatment while improving potato yield, providing a strong basis for biocontrol utilization in crop production.
\end{abstract}

Keywords: soil microbial communities; microbial diversity; biocontrol; potato

\section{Introduction}

Plants serve as a niche for growth and proliferation of a diversity of microorganisms, including bacteria, fungi, protists, nematodes, and viruses (the plant microbiota). These microorganisms form complex co-associations with plants and have important roles in promoting their health and productivity in natural environments. Complex plant microbial communities are comprised of taxa from diverse phyla that belong to several lineages. In recent years, culture-independent high-throughput sequencing has greatly expanded the number of microorganisms known to reside in and on plants as well as in the surrounding environments [1-3]. Among the plant-associated microbiota, bacteria and to lesser extent fungi are the most dominant forms and are relatively well-studied compared with other members of the community, but other groups-such as archaea, algae, nematodes, and protists-also have important roles in plant health and productivity [4,5]. Several genes that govern plant interactions with the associated microbiomes have been identified and characterized, which have increased our understanding of how microorganisms adapt to and modulate the plant environment [6,7]. The members of a plant microbiota comprise beneficial, neutral, and pathogenic microorganisms. Microbial communities associated with their hosts have been shown to promote plant growth, nutrient uptake, and resistance to pathogens $[8,9]$. However, the molecular mechanisms that govern plant-microbe interactions at a community level are still not well understood. To achieve a more comprehensive understanding, it will be necessary to first characterize the mechanisms that drive the 
assembly of plant-associated microbiomes in the rhizosphere. Second, the biochemical and genetic features of the microorganism-microorganism and host-microorganism interactions that result in beneficial ecological outcomes need to be identified. Such data will inform the design and construction of functional microbial systems de novo that are based on predictive models of plant-microbiome interactions. Furthermore, the development of microbial inoculants, signaling compounds, and other tools will enhance the understanding of microbiome function in agricultural ecosystems.

Soil microorganisms, which closely interact with plants, are an important factor affecting plant health [2]. The emergence and propagation of plant pathogens that cause plant diseases is well documented, but in contrast, soil microorganisms that can prevent plant diseases and inhibit pathogens to maintain plant health are only now being identified for commercial exploitation [10]. In the last decade, an increasing number of studies have focused on the soil microbial communities involved in plant disease prevention and treatment [11-13]. Disease occurrence is often accompanied by changes in the microbial community. Therefore, additional efforts to rehabilitate the microbial community may be more effective in curing plant diseases than simply controlling pathogen populations $[14,15]$. Using microorganisms to control plant diseases has been successful under laboratory and greenhouse environments, but biocontrol, considered a highly desirable approach for controlling soil-borne diseases, has not been as successful in the field as initially predicted $[10,16,17]$. The effective use of biological control agents is potentially an important component of sustainable agriculture. There has been an increased interest in using combinations of biological control agents to exploit the potential synergistic effects among them.

Biocontrol agents are microorganisms that exert harmful effects on pathogens, thereby improving plant health [18]. Biocontrol agents can establish beneficial relationships with various plant species by direct or indirect mechanisms $[18,19]$. Entomopathogenic fungal endophytes could function as biocontrol agents to prevent pests and diseases as well as promote plant growth [20]. Moreover, Bacillus strains exhibit their biocontrol capacity predominantly through inhibitory activity on the growth of plant pathogens as well as inducing systemic resistance in plants and competing for ecological niches with plant pathogens [21]. For example, yeasts and bacteria of the genus Bacillus serve as biological control agents of Athelia (Sclerotium) rolfsii through antagonistic interactions [22]. Bacterial endophytes suppress stripe rust infection and enhance wheat yields, which can be exploited as potential biocontrol agents of wheat rusts [23]. The bacterial isolates BETS11 and BETR11 recovered from surface-sterilized root, stem, and leaf tissues of tomato may be used as efficient biofertilizer and bio-control agents for tomato production in the island agricultural ecosystem [24]. The current investigation mainly focused on the modifications of the soil microbiome brought about by the application of a biocontrol agent in the potato production system. Several functional mechanisms of biocontrol were delineated.

Potato (Solanum tuberosum) is an important crop that is cultivated under a variety of geographic locations and climatic conditions. The yield and tuber quality are determined by both biotic and abiotic factors, such as drought, nitrogen, and phosphorous status in the soil and microbiome in potato rhizosphere [25-28]. Our previous study demonstrated that Bacillus subtilis strain Bv17 could effectively colonize the rhizosphere of host plants and significantly reduce the propagation and expansion of Verticillium dahliae in the plants; however, the functional mechanisms by which $B$. subtilis strain Bv17 brings about these changes is still unknown [29]. Therefore, we used the Bacillus subtilis strain Bv17 to treat soil prior to planting potato, which is followed by periodic soil sampling for ITS and $16 \mathrm{~S}$ sequencing to determine the dynamics of abundance and composition of soil microbial communities. Thus, our aim was to detect the influence of biocontrol of Bacillus subtilis strain Bv17 on microbial communities in soil and discover the relationship between microbial communities and quality of potatoes after biocontrol strain treatment. Results showed that microbial community in soil changed by Bacillus subtilis strain Bv17 treatment. 
The yield of potato also significantly increased, providing a strong basis for biocontrol utilization in crop production.

\section{Results}

\subsection{Experimental Design and Treatment Structure}

Bacillus subtilis strain Bv17 was safe and non-toxic to host plants, but it could effectively colonize the rhizosphere of host plants and significantly reduce the propagation and expansion of $V$. dahliae in the plants [29]. To test the efficacy of B. subtilis strain Bv17 and to analyze the microbial community between the rhizosphere soil and the plant, potatoes were planted in a field in Shandong province located in the eastern part of China in plots treated with the B. subtilis strain Bv17 (DT), while those treated with water served as the untreated control (DC). Soil samples were collected at two-month intervals three times (D1C, D1T, D2C, D2T, D3C, and D3T), and potatoes were harvested at crop maturity (PC and PT) (Figure 1A). A total of 90 soil samples were collected and divided into six groups (D1C, D1T, D2C, D2T, D3C, and D3T) with 15 samples in each group. All of the soil samples were used for ITS and $16 \mathrm{~S}$ sequencing to analyze the microbial community. Furthermore, a total of 60 potato samples were collected at crop maturity with 30 each coming from plots treated with Bacillus subtilis strain Bv17 and from the untreated control. The skin of potatoes was collected used for ITS and 16S sequencing to investigate the microbial communities between the control and B. subtilis strain Bv17-treated samples. In addition, potato tubers were used for the detection of qualitative and quantitative index, such as weight, dry matter content, starch content, and others.

\subsection{Data Characteristic of Soil Samples}

Total DNA from all 90 soil samples were extracted and sent for $16 \mathrm{~S}$ and ITS sequencing to a depth of more than $30 \mathrm{Mb}$ each. Each group (D1C, D1T, D2C, D2T, D3C, and D3T) contained fifteen soil samples (Supplementary Table S2). The average numbers of raw DNA sequence data for each soil sample of bacteria were about 70,000 reads, and approximately $99 \%$ of the reads from each sample remained after filtering for quality and size (Supplementary Table S3). While the number of reads for ITS was lower than for 16S, the read utilization ratio ranged between $84 \%$ and $99 \%$ (Supplementary Table S4). Paired end reads were spliced into tags through the overlapping relationship between reads. A total of 5,617,159 tags were obtained for all $16 \mathrm{~S}$ samples, with an average of 62,412 tags per sample, with an SD value of 2816. The average tag length was $268 \mathrm{bp}$, and the SD value was $23 \mathrm{bp}$ (Supplementary Table S5). For ITS, there was a total of 5,438,323 tags for all samples combined after removing the primers. On average, each sample was 60,425 tags, with an SD value of 2930, and an average length of $219 \mathrm{bp}$, with an SD value of 22 (Supplementary Table S6). The clean tags were subsequently clustered into different operational taxonomic units (OTUs) at $97 \%$ similarity. A preliminary assessment of the abundance of OTUs suggested the species richness of the samples. A total of 5631 OTUs were identified from the 16S data, and 2236 OTUs were identified from the ITS data (Supplementary Table S1). The number of observed bacterial OTUs was much greater for the soil samples than fungal OTUs (Supplementary Table S1).

\subsection{Analysis of the Microbial Community Composition between Treated and Untreated Soils}

A comparative analysis of the microbial community composition between different sample groups was made to investigate whether there was an association between the treatments and changes in the microbiome. Principal component analysis (PCA) was used to construct 2D graphs to summarize the factors mainly responsible for differences in the OTU composition. The OTUs of bacterial community compositions clustered together (Figure 2A). Bacteria of D1C and D1T were different from D2C, D2T, D3C, and D3T, while there were no measurable differences in their community compositions between the four subsequent samples (Figure 2A). Bacterial communities in D2T and D3T were more closely clustered than in the control treatments, D2C and D3C (Figure 2A). However, obvious 
distinctions in community compositions were found among D1C, D1T, D2C, D2T, D3C, and D3T for fungi (Figure 2B). The differences in the community composition of fungi in D1T with D1C was smaller than variations observed between D2T and D3T, D2C and D3C (Figure 2B).

A

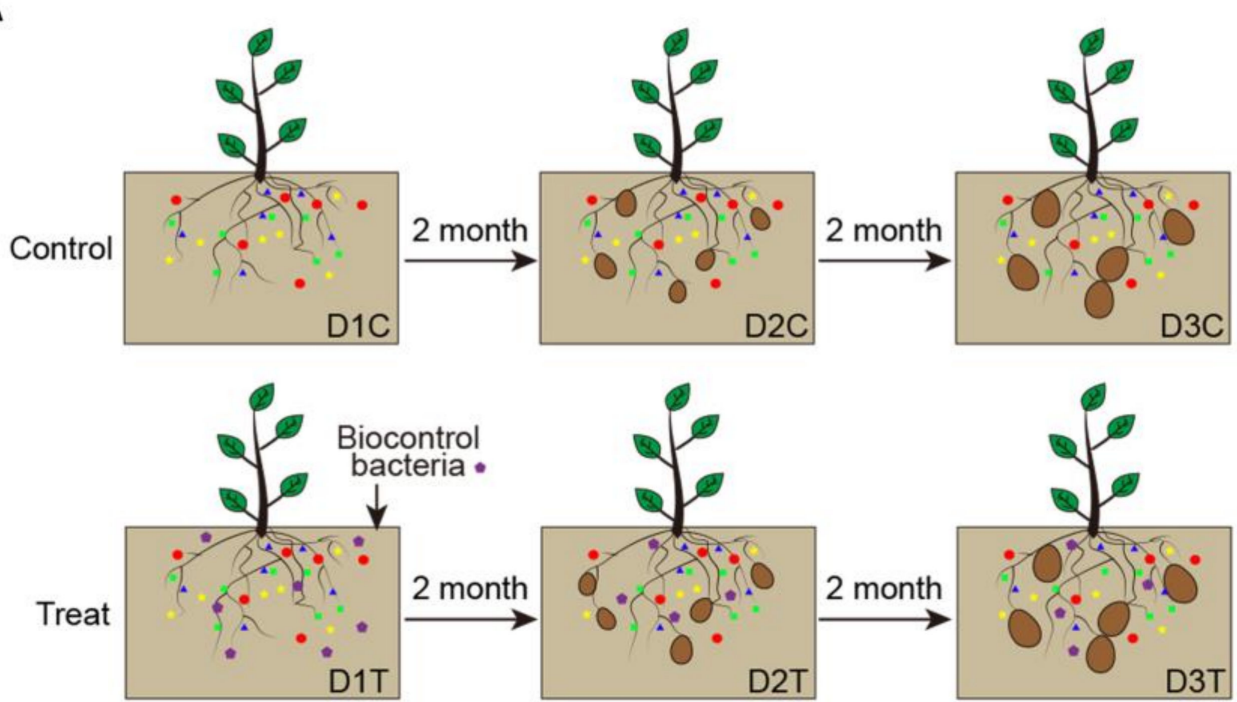

B

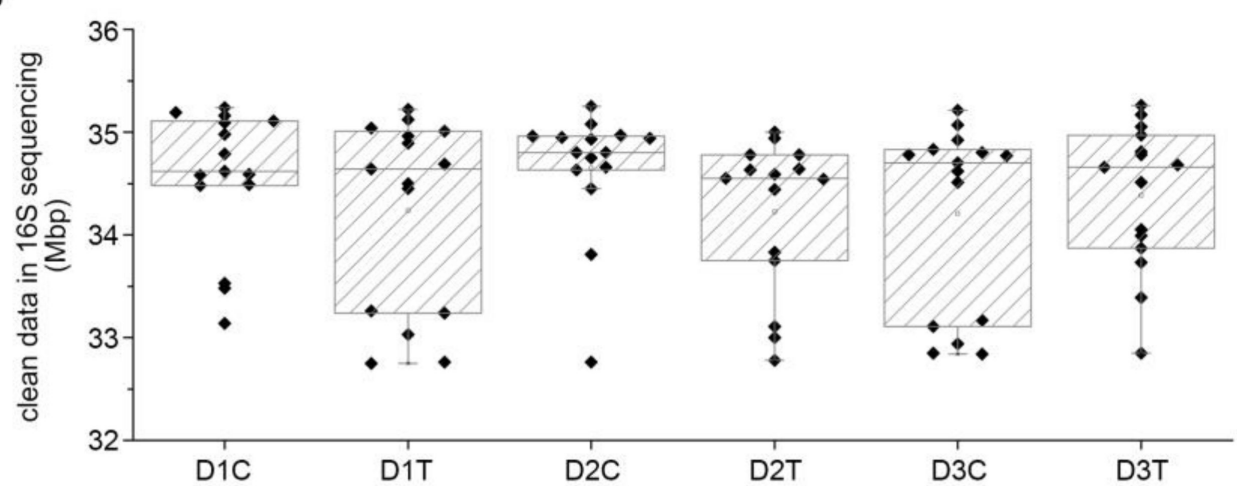

C

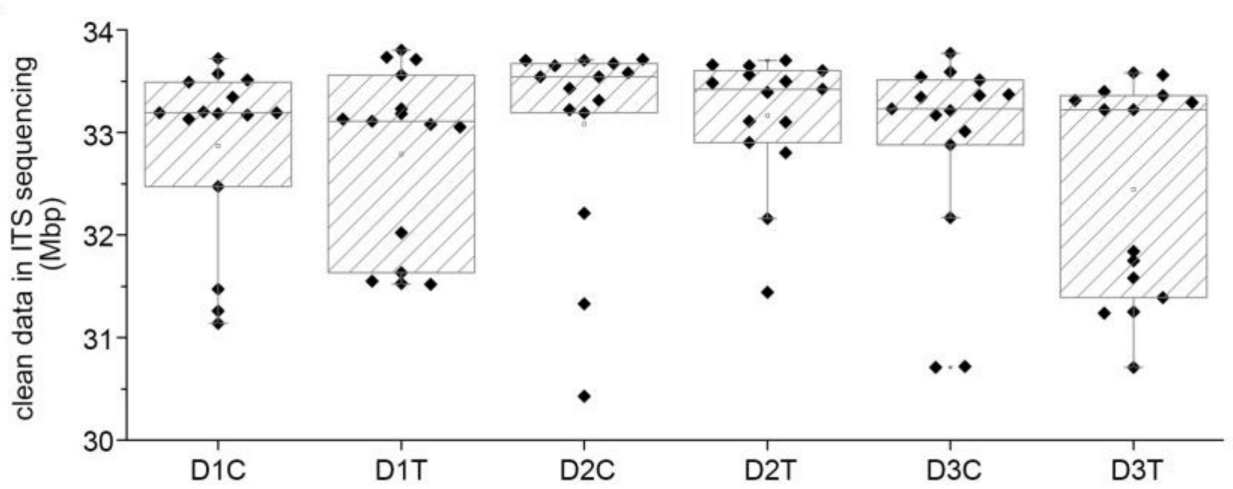

Figure 1. Characteristic of biocontrol treatment experiment and high-through sequencing. (A) Schematic diagram of experimental design. (B) The depth of $16 \mathrm{~S}$ sequencing for each group of soil samples. (C) The depth of ITS sequencing for each group of soil samples. 
A

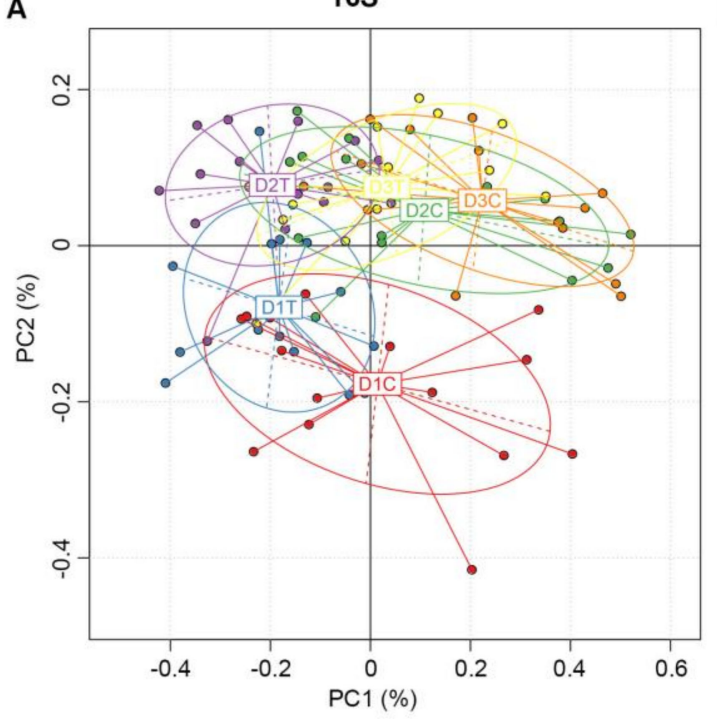

B

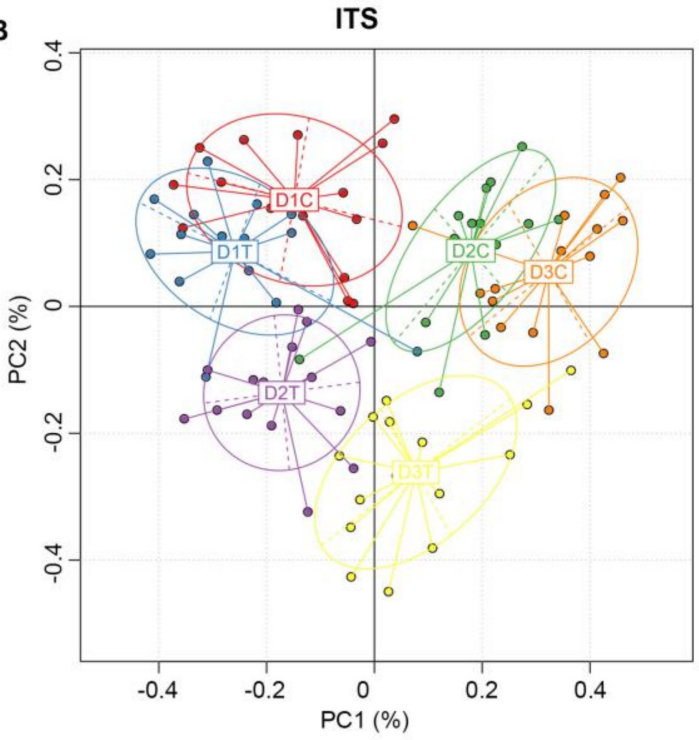

Figure 2. Principal component analysis based on OTU abundance in bacteria (A) and fungi (B). X-axis represents the mean principal component 1 and Y-axis represents the mean principal component 2. Numbers within parentheses represents contributions of principal components to differences among samples. Each dot represents a soil sample, and different colors represent different groups.

\subsection{Microorganism Community Diversity in Soil Samples under Different Conditions}

The identified OTUs were distributed across 35 bacterial phyla (Figure 3A). The predominant phyla were Proteobacteria and Actinobacteria, which together accounted for about $66 \%$ of the population, followed by Acidobacteria with $10.15 \%$ of OTUs (Figure 3A). Firmicutes, Chloroflexi, WPS-2, Bacteroidetes, Gemmatimonadetes, and TM7 were recovered with a small proportion at the phylum level (Figure 3A). Proteobacteria was enriched highest in D3T among all six groups, especially compared with D3C (Figure 3A). The level of Acidobacteria was significantly higher in DT compared with DC, while the level of Actinobacteria was significantly less in DT than DC (Figure 3A). Furthermore, the bacteria detected corresponded to 31 different genera, without no significant enrichment, including Acinetobacter, Bacillus, Leptothrix, Cupriavidus, Rhodoplanes, and others (Figure 3B). The enrichment of several bacteria was reduced in DT compared with DC, and these included Cryocola, Geodermatophilus, and Alicyclobacillus (Figure 3B).

For the fungal kingdom, the OTUs spanned 13 phyla and 65 genera (Figure 4). From these, Ascomycota and Basidiomycota comprised 90\% (Figure 4A). Interestingly, Ascomycota and Basidiomycota were both higher in natural soil (D1C and D1T) but reduced in D2T and D3T compared with D2C and D3C, respectively (Figure 4A). Alternaria, Fusarium, and Humicola formed the major proportion at the genus level (Figure $4 \mathrm{~B}$ ). The application of $B$. subtilis strain Bv17 suppressed Alternaria and Humicola (Figure 4B). These results indicated that the microbial distribution differed between soils treated with B. subtilis strain Bv17 and untreated soils.

\subsection{Similarities and Differences of OTUs between Six Groups of Soil Samples}

The number of common and unique OTUs of multiple samples is shown in a Venn diagram. For bacteria, there were 2840 common OTUs among D1C, D2C, and D3C, and 3313 OTUs in D1T, D2T, and D3T. There were 2476 common OTUs between DC and DT, and 364 and 837 of these OTUs were unique to DC and DT, respectively (Figure 5A and Supplementary Figure S1). A total of 4920 OTUs were discovered in DC (D1C, D2C, and D3C) and 5191 OTUs were discovered in DT (D1T, D2T, and D3T). Among the 4480 common to DC and DT, 440 were unique in DC and 711 were unique in DT (Figure 5B and Supplementary Figure S1). These 711 specific OTUs in DT corresponded to differential 
abundance of related bacteria (Supplementary Table S7). For fungi, there were 935, 892, and 760 common OTUs between D1C and D1T, D2C and D2T, and D3C and D3T, respectively (Supplementary Figure S2). There were 156 unique OTUs in DC, 224 unique OTUs in DT, and 559 common OTUs between DC and DT (Figure 6A). Finally, a total of 342 unique OTUs were found in DC (D1C, D2C, and D3C) and 503 unique OTUs were found in DT (D1T, D2T, and D3T), with 1391 common OTUs between DC and DT (Figure 6B). The 503 unique OTUs in DT corresponded to the differential abundance of related fungi (Supplementary Table S8).

A

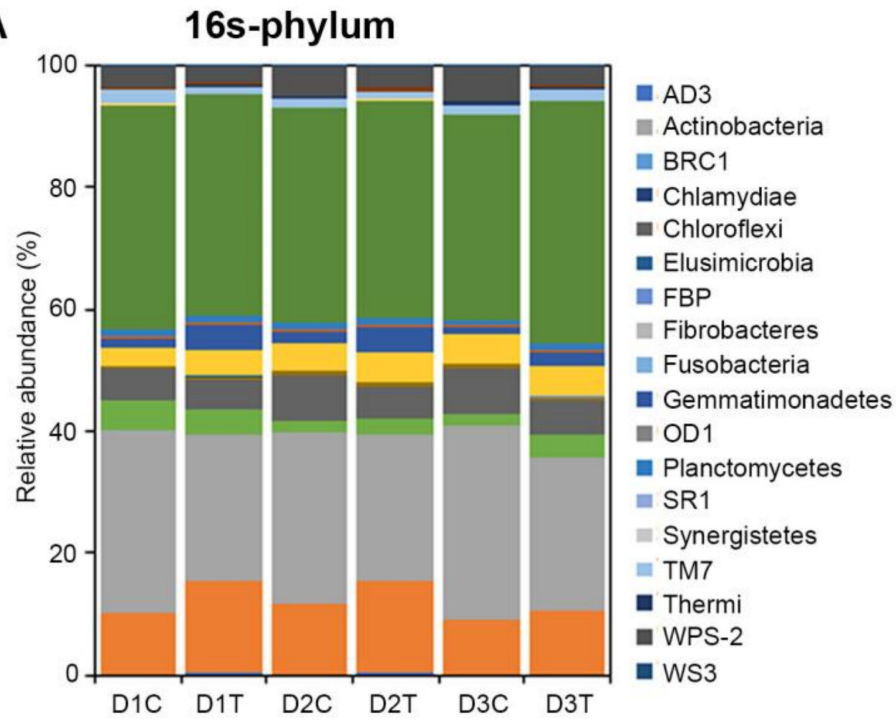

\author{
- Acidobacteria \\ narmatimonadetes \\ Bacteroidetes \\ - Chlorobi \\ - Cyanobacteria \\ - Euryarchaeota \\ - FCPU426 \\ 들 Firmicutes \\ anO2 \\ - Nitrospirae \\ - OP3 \\ - Proteobacteria \\ - Spirochaetes \\ - TM6 \\ = Tenericutes \\ - Verrucomicrobia \\ WS2
}

\title{
B
}

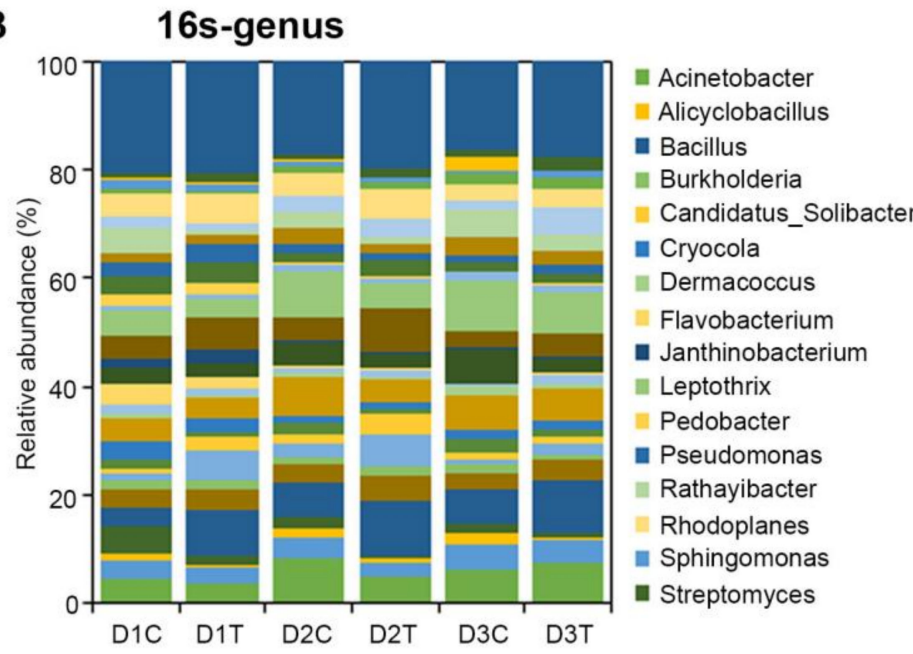

Figure 3. Relative abundances (percentage of sequences) of bacteria at phylum (A) and genus (B) levels in each group.

Ace, Chao, Shannon, and Simpson indices were used to analyze the richness and diversity of the soil microbial community. The Chao and Ace in DT were higher than in DC both for bacteria and fungi (Supplementary Table S9). The value of Shannon and Simpson were small; Shannon was higher in DT than in DC, but Simpson was lower in DT than in DC without significance (Supplementary Table S9). Particularly, the Chao index and Shannon index of the soil samples were selected to show the detailed distribution in each group. The results showed that both the Chao and Shannon index were relatively stable among D1T, D2T, and D3T, while the Chao and Shannon were decreased from D1C to D3C similarly in both bacterial and fungal communities (Figure 5C,D and Figure 6C,D), which 
suggested a higher diversity of the bacterial and fungal community in the soil treated by $B$. subtilis strain Bv17.

\section{A}

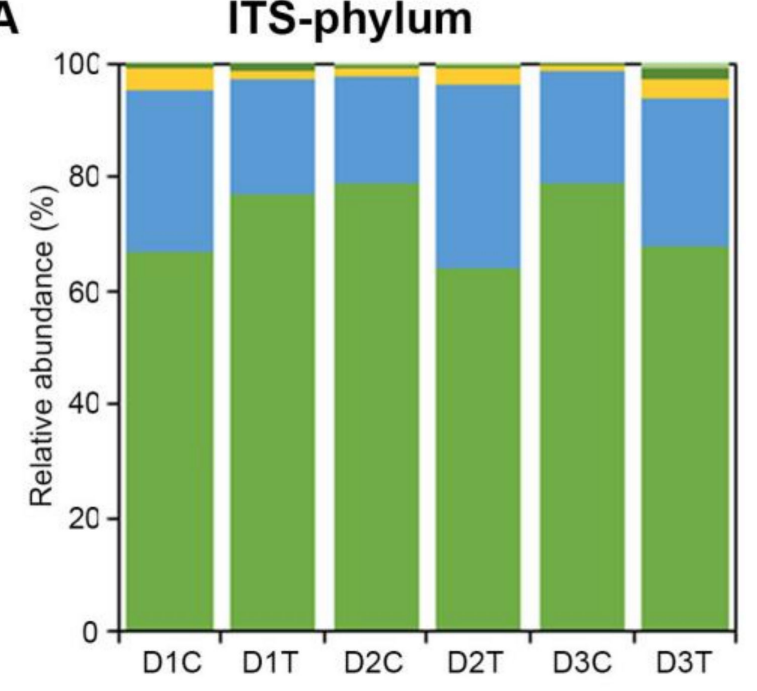

- Ascomycota

- Basidiomycota

in Cercozoa

- Chytridiomycota

- Cnidaria

- Entomophthoromycota

= Glomeromycota

- Kickxellomycota

Mortierellomycota

- Mucoromycota

- Olpidiomycota

- Rotifera

nozellomycota

\section{B}

\section{0}

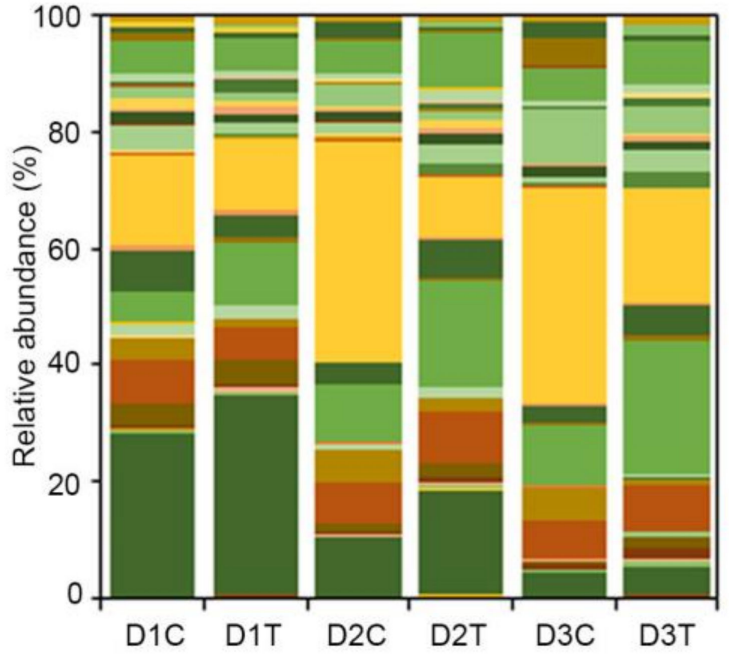

Acaulium

- Alternaria

- Bullera

- Chrysosporium

- Conocybe

Echria

- Gibberella

- Hyphopichia

Microscypha

= Oidiodendron

n Pseudogymnoascus

= Septoria

n Trichothecium

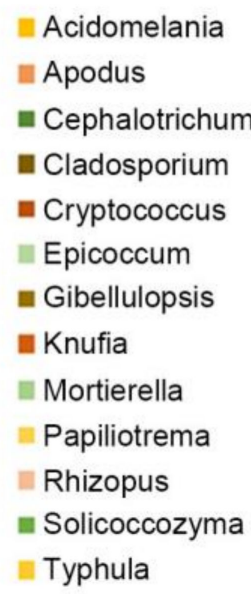

acremonium

-Actinomucor

Agrocybe

Arthrobotrys

aspergillus

- Chaetomium

Boothiomyces

=Chloridium

aClitopilus

In Coniophora

Conlarium

ECurvularia

- Cyphellophora

Dioszegia

Exophiala

arlobasidium

Eusarium

- Guehomyces

= Hannaella

numicola

- Leptosphaeria

- Melanospora

microascus

anucor

=Penicillium

Myrmecridium

ENaganishia

aPhaeosphaeria =Pichia

Rhodotorula

saitozyma

n Sarocladium

- Talaromyces

n Trechispora

- Trichoderma

athers $(<0.5 \%)$

Figure 4. Relative abundances (percentage of sequences) of fungi at phylum (A) and genus (B) levels in each group. 
A

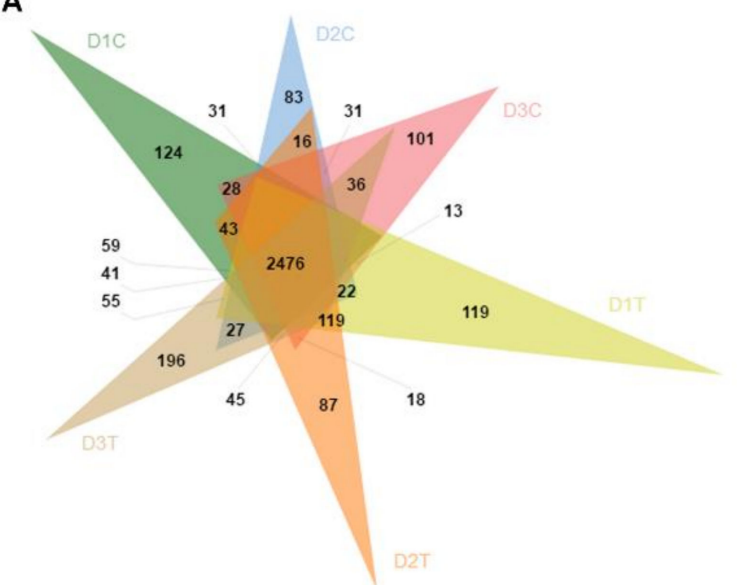

B

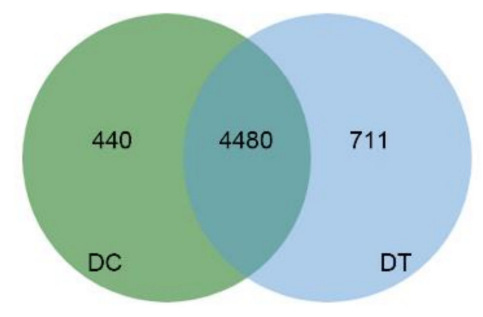

C

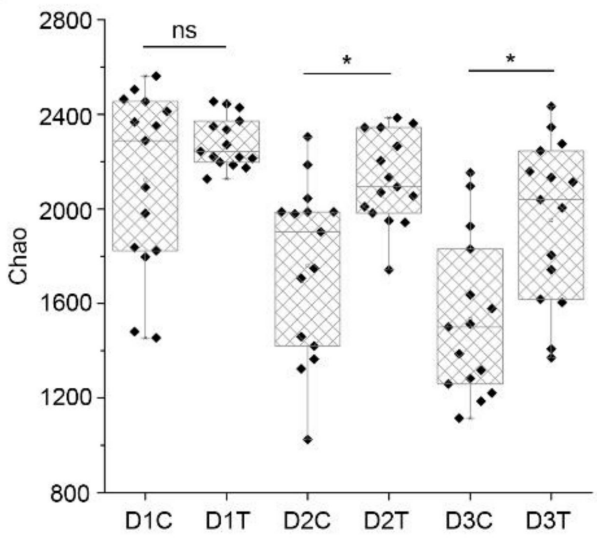

D

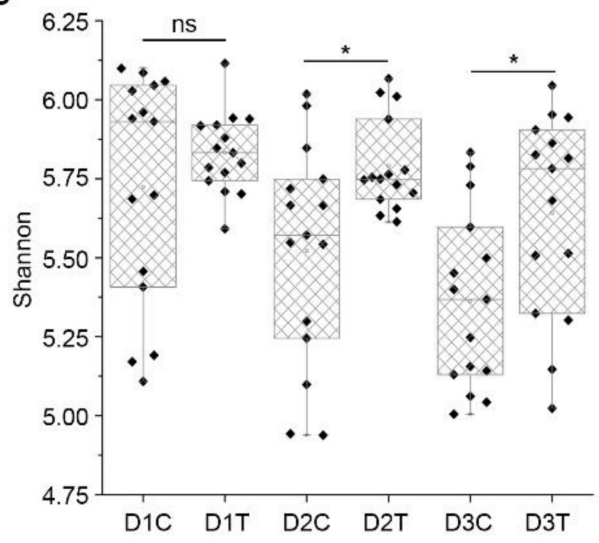

Figure 5. Analysis of bacterial diversity in soil samples. (A) Venn diagram of OTUs between each soil group in bacteria. DT refers to soil samples derived from plots treated with Bacillus subtilis strain Bv17, and those that came from plots treated with water are labeled DC. Soil samples were collected at two-month intervals three times (D1C, D1T, D2C, D2T, D3C, and D3T). (B) Venn diagram of OTUs between DC and DT. (C) The box diagram of alpha diversity of the Chao index in each soil groups. (D) The box diagram of alpha diversity of the Shannon index in each soil groups. Asterisks indicate statistically significant differences at $p<0.05$ according to pairwise $t$-tests, and ns means no significant difference between treatments.

\subsection{Analysis of Bacteria Diversity of Potato by $16 S$ Sequencing}

The number of raw DNA sequence data and clean reads data for each potato sample of bacteria was about 70,000 reads, and more than 99\% of the reads from each sample remained after filtering for quality and size (Supplementary Table S10). The number of OTUs was annotated at $97 \%$ similarity to the cluster. A total of 257 OTUs were produced among 16S from potato samples (Supplementary Table S11). The average coverage was more than 99\%, and the index of alpha diversity, including Chao, Ace, Shannon, and Simpson from PT was not significantly different from PC (Supplementary Table S11). The microorganism community similarity in different samples of potato was determined by PCA. The bacterial community in PC appeared more similar, but the three samples of PT clustered separately from each other (Figure 7A). The bacterial OTUs were assigned into 17 phyla and five genera, after combining the species with abundance less than $0.5 \%$ into others. The dominant bacterial phylum across all samples was Cyanobacteria, accounting for $>96 \%$ of the OTUs. The second dominant bacterial phylum across all samples was Proteobacteria, with nearly $3.5 \%$ in PC and $2.6 \%$ in PT (Figure $7 \mathrm{~B}$ ). The assigned five genera were Agrobacterium, Leptothrix, Ochrobactrum, Ralstonia, and Sediminibacterium, comprising 
about $40 \%$ (Figure 7C). Furthermore, the OTUs were integrated among PC (PC1 and PC2) and PT (PT1 and PT2). A total of 143 OTUs were discovered in PC, and 244 OTUs were found in PT. From these OTUs, 130 were matched both in PC and PT, 13 were unique in PC, and 114 were unique in PT (Figure 7D). The unique OTUs in PT were significantly higher than in PC, and the unique OTUs in PT were annotated into bacteria species (Supplementary Table S12).

A

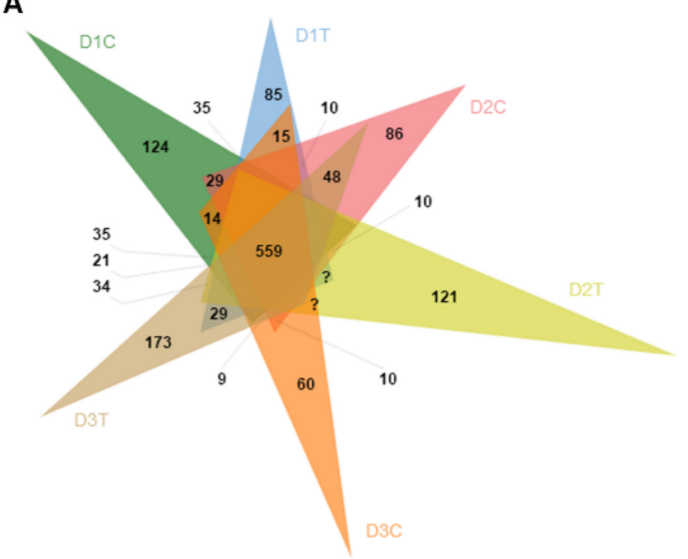

C

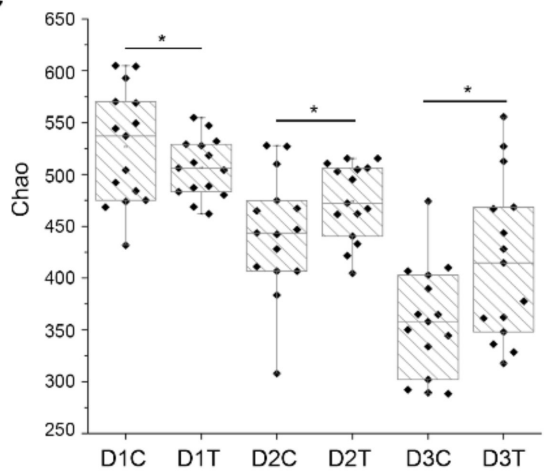

B

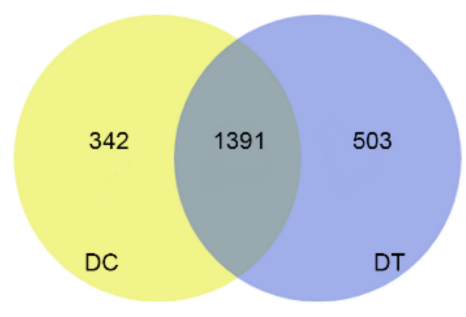

D

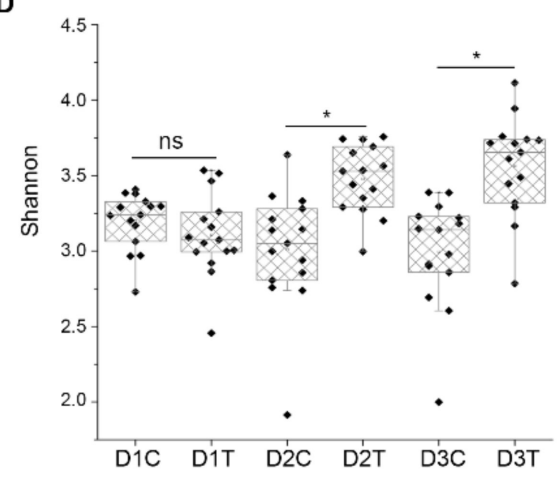

Figure 6. Analysis of fungal diversity in soil samples. (A) Venn diagram of OTUs between each soil groups in fungi. DT refers to soil samples derived from plots treated with Bacillus subtilis strain Bv17, and those that came from plots treated with water are labeled DC. Soil samples were collected at two-month intervals three times (D1C, D1T, D2C, D2T, D3C, and D3T). (B) Venn diagram of OTUs between DC and DT. (C) The box diagram of alpha diversity of Chao index in each soil group. (D) The box diagram of alpha diversity of the Shannon index in each soil groups. Asterisks indicate statistically significant differences at $p<0.05$ according to pairwise $t$-tests, and ns means no significant differences between treatments.

\subsection{Analysis of Potato Quality Measures}

Bacillus subtilis strain Bv17 treated plants had a higher tuber weight compared with the untreated control (Figure 8A). The internal quality of potato tubers is often estimated by the starch, sugar, and protein content [30]. For the processing industry and cooking purposes, the accepted dry matter content is between $18 \%$ and $20 \%$ [30]. The dry matter content in PC was $18.4 \%$ lower than in PT (20.6\%) (Figure 8B). In addition, the content of starch, protein, and reducing sugars was also higher in PT compared to tubers in PC (Figure 8C-E). Potato tubers are an important source of vitamin $C$, which serves as an antioxidant with healthpromoting effects [30]. The vitamin C levels in PC and PT groups were $48.4 \mathrm{mg} / 100 \mathrm{~g}$ and $48.5 \mathrm{mg} / 100 \mathrm{~g}$, respectively, and these were not statistically different (Figure 8F). 
A

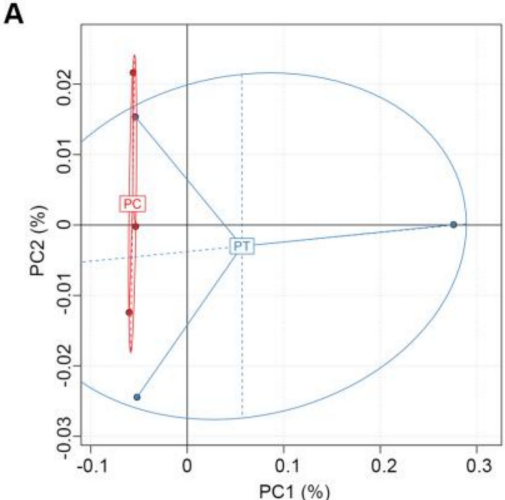

C

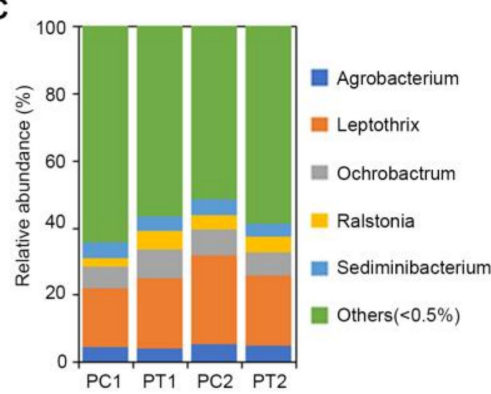

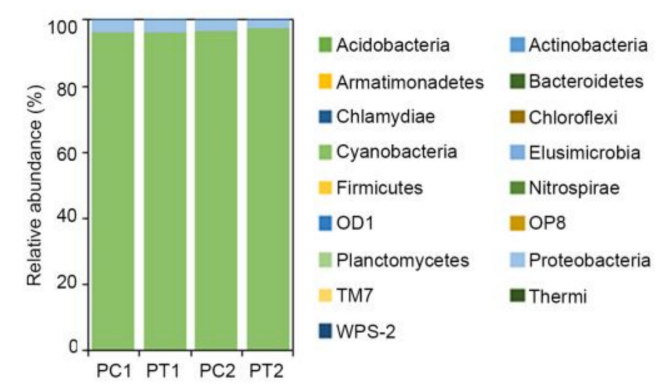

D

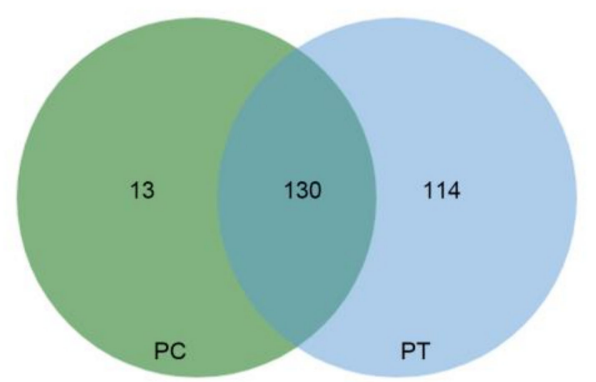

Figure 7. Characteristics of $16 \mathrm{~S}$ sequencing data of potato samples. (A) Principal component analysis based on OTU abundance in bacteria. X-axis represents principal component 1 and Y-axis represents principal component 2. Numbers within parentheses represent contributions of principal components to differences among samples. Each dot represents an individual soil sample, and different colors represent different groups. (B) Relative abundances (percentage of sequences) of bacteria at the phylum level. (C) Relative abundances (percentage of sequences) of bacteria at the genus level. (D) Venn diagram of OTUs between PC and PT. PC refers to potatoes derived from the control treatment, and PT refers to potatoes derived from plots treated with Bacillus subtilis strain Bv17.

A

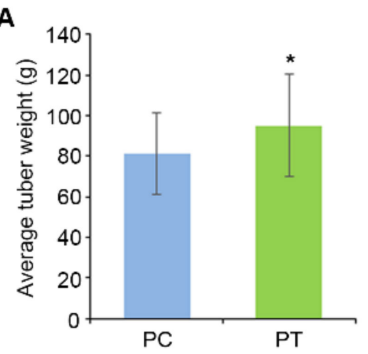

D

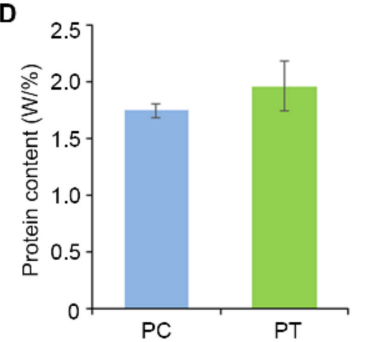

B

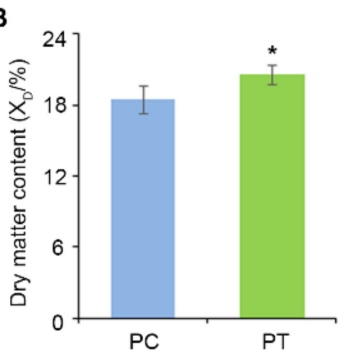

E

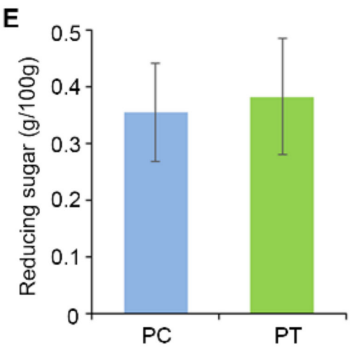

C
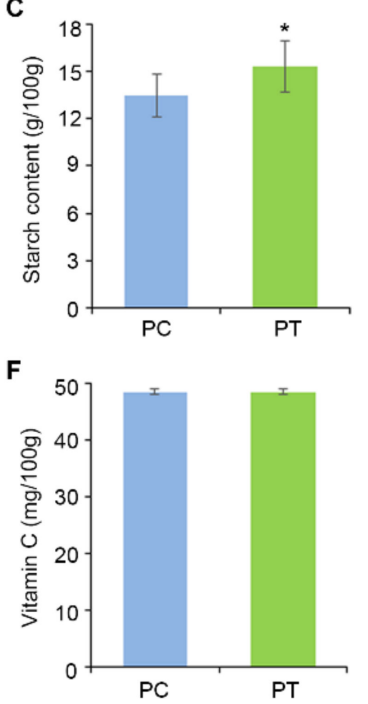

Figure 8. Effect of biocontrol treatment on potato tuber yield and quality. (A) Bar chart of average tuber weight. $n=18$ (B) Bar chart of dry matter. (C) Bar chart of starch content. (D) Bar chart of protein content. (E) Bar chart of reducing sugar content. (F) Bar chart of vitamin $C$ content. Asterisks indicate statistically significant differences at $p<0.05$ according to $t$-test. 


\section{Discussion}

Several studies have demonstrated that the application of biocontrol agents could influence the microbial community. For example, the application of Pseudomonas fluorescens $p c 78$ to the soil affected the microbial community structure in the tomato rhizosphere [31]. Similarly, the endophytic microbial abundance was significantly higher in the stem of maize grown in Trichoderma asperellum granules-treated soil, with reductions in deoxynivalenol (DON) and fumonisin B1 (FB1) accumulation [32]. The application of biocontrol can influence seasonal epiphytic microbial dynamics on grapevine leaves [33]. In our research, we found that the abundance of microbial community of soil changed after Bacillus subtilis strain Bv17 treatment. Soil samples from DC and DT showed no statistically significant differences in the number of bacteria and fungi (Figure 1B,C). Interestingly, the OTU numbers of both bacteria and fungi from D1C to D3C progressively decreased, but the number of OTU from D1T to D3T registered an increase (Supplementary Figures S1 and S2, and Supplementary Table S9). In addition, the Chao and Ace indices for DT were higher than in DC both for bacteria and fungi (Supplementary Table S9). The values of Shannon and Simpson indices were small, with Shannon being higher in DT than in DC. In contrast, Simpson was lower in DT than in DC, and the differences were not significant (Supplementary Table S9). These results indicated that the bacterial and fungal richness (Ace and Chao) were markedly increased after the Bacillus subtilis strain Bv17 treatment; however, the differences were not statistically significant based on the Shannon and Simpson indices.

The microbiome outside of plant tissues performs various plant beneficial activities such as suppression of potential phytopathogens and promotion of plant growth. The microbiome of plants plays a crucial role in both plant and ecosystem health [34]. In the soil, high microbial activity reflects the intensity and direction of various biochemical activity and the direction of various biochemical reactions. Even a slight decrease in soil microbial diversity or a change in the structure and function could affect the availability and absorption of nutrients [35]. The rhizosphere microbiome is important for plant growth and health [36,37]. Plants can recruit protective microorganisms to enhance microbial activity to suppress pathogens in the rhizosphere upon pathogen or insect attack [36,37]. The research showed that the continuous cropping of maize seed production could increase pathogenic pathogens, making maize in danger of pathogen invasion [38]. Here, the changes in the soil microbiol community following the application of B. subtilis strain Bv17 affected potato tuber growth (Figure $8 \mathrm{~A}$ ). Whether the impacts on potato plant and tuber health were influenced by decreasing the harmful effects of pathogens or if the microbial enhancements of the rhizosphere environment brought about the changes in potato is unclear and teasing out these differences is currently underway.

The biocontrol of soilborne plant diseases in cultivated crops has been explored more intensely in recent years. P. dispersa strains were able to inhibit black rot in sweet potato plants [39]. The use of a combination of biocontrol strains as a potential strategy could limit the soft rot and blackening diseases caused by D. dianthicola on potato plants and tubers [40]. Streptomyces violaceusniger AC12AB could promote potato growth by decreasing potato common scab and increasing potato yield [41]. The effect of Pseudomonas fluorescens, Bacillus subtilis, P. aeruginosa, and Trichoderma spp. to enrich the growth and yield of potato crop and induce resistance against wilt disease caused by Ralstonia solanacearum [42]. The application of a beneficial microbial combination efficiently enhanced plant and soil health under biotic stress through improving the microbial community structure [43]. Lactobacillus plantarum SLG17 and Bacillus amyloliquefaciens FLN13 function as biocontrol agents on durum wheat at a stage from heading until anthesis against Fusarium spp. [44]. In our research, we found that the yield and quality of potato were correspondingly improved by Bacillus subtilis strain Bv17 treatment. The important quality traits of the potato tubers such as the starch, sugar, protein, and vitamin $C$ contents improved in biocontrol treated plots (Figure 8), even though the bacterial community on potato was unaltered in treated and untreated plots. 


\section{Materials and Methods}

\subsection{Preparation and Dosage of Bacillus subtilis Strain Bv17 in Field}

Bacillus subtilis strain Bv17 was cultured in LB medium at $37^{\circ} \mathrm{C}$ for $48 \mathrm{~h}$ on a rotating shaker at $200 \mathrm{rpm}$. Bacillus subtilis strain Bv17 fermentation liquid was added to $1 \mathrm{~kg}$ of carrier diatomite until the diatomite was saturated. This mixture was air-dried and pulverized to obtain a finely powdered product with an adsorption capacity of $1.2 \mathrm{~L} / \mathrm{kg}$. Prior to the application of the product in the field, a wetting agent (sodium dodecyl sulfonate, sodium lignosulfonate) and a dispersant (sodium carboxymethyl cellulose) were added to obtain a wettable powder. Two kg wettable powder was evenly spread on $666.67 \mathrm{~m}^{2}$ and tilled in to serve as the biocontrol treatment. An area of equal size was treated with water, and it served as the untreated control.

\subsection{Soil Treatment and Collection of Soil and Potato Sample}

Potato was planted in Shandong province $\left(37^{\circ} 45^{\prime}\right.$ north latitude, $116^{\circ} 29^{\prime}$ east longitude, altitude $27 \mathrm{~m}$ ), which is in the east of China. The average rainfall was $466 \mathrm{~mm}$, and the average temperature was $14.5^{\circ} \mathrm{C}$ in 2019. The experiment included two main treatments. The first included the application of Bacillus subtilis strain Bv17 to the soil prior to planting, and the second was treated with water to serve as untreated control. Potato seedings were planted on 24 February 2019. The row spacing of potatoes was $40.0 \mathrm{~cm}$, and the plant spacing was $37.5 \mathrm{~cm}$. The plot area was $72.0 \mathrm{~m}^{2}(9.0 \mathrm{~m} \times 8.0 \mathrm{~m})$. The samples of soil were named D1C (Control) and D1T (Treat). Samples were collected approximately every two months from each treatment (14 April 2019, and 8 June 2019), and these were named D2C/D2T and D3C/D3T, respectively. Fifteen samples were collected from the treated and untreated plots at each sampling for a total of 90 samples during the cropping cycle. Twenty potatoes were collected at crop maturity (8 June 2019) from treated (PT) and untreated control (PC) plots. Collected potatoes were used for $16 \mathrm{~S}$ sequencing and determining quality parameters as described below.

\subsection{DNA Extraction and PCR Analysis}

The DNAsecure Plant Kit (Tiangen, Beijing, China) was used to extract the genomic DNA from the soil samples according to the manufacturer's instructions. The final DNA elution was performed using sterile deionized water. DNA quality and quantity were measured by NanoDrop1000 (Thermo Fisher Scientific, Inc., Newark, DE, USA) and by agarose gel electrophoresis. Extracted DNA was stored at $-80^{\circ} \mathrm{C}$. The V3V4 region of the $16 \mathrm{~S}$ rRNA gene was amplified using the primer V3V4-F: ACTCCTACGGGAGGCAGCA and V3V4-R: GGACTACHVGGGTWTCTAAT. For fungal community analysis, the ITS sequence was amplified with primer ITS1-F: GGAAGTAAAAGTCGTAACAAGG and ITS1-R: GCTGCGTTCTTCATCGATGC.

\subsection{Sequencing Analysis}

Paired-end reads were generated with the Illumina HiSeq2500 platform. The raw reads were preprocessed by removing the adapter, ambiguous bases, and those with low complexity to obtain the clean reads [45]. For a pooling library with barcode samples mixed, the clean reads were assigned to corresponding samples by allowing 0 base mismatch to barcode sequences with in-house scripts. Then, the consensus sequences were generated by FLASH (Fast Length Adjustment of Short reads, v1.2.11) [46]. The tags were clustered to the Operational Taxonomic Units (OTUs) by using scripts from software USEARCH (v7.0.1090) [47]. OTUs were filtered by sequences that were unassigned and not assigned to the target species. The filtered OTUs were used for downstream analyses. Alpha diversity was calculated to determine the complexity of species diversity for individual samples through several indices, including observed species, Chao, Ace, Shannon, and Simpson [48]. The indices were calculated by Mothur (v1.31.2). The formulae for the calculation of each index can obtained at http:/ / www.mothur.org/wiki/Calculators (accessed on 14 September 2021). 


\subsection{Detection of Quality of Potato Tubers}

From the Bacillus subtilis strain Bv17 treated, and water treated plots, 18 representative potato tubers were collected to calculate the weight of each tuber [49]. Samples of potato tubers were drying at $105^{\circ} \mathrm{C}$ for $30 \mathrm{~min}$ and then at $80^{\circ} \mathrm{C}$ to constant weight [49]. Furthermore, the starch content, protein content, reducing sugar content, and vitamin $\mathrm{C}$ content were determined following the procedures in the literature [30].

\subsection{Statistical Analysis}

Analysis of variance (ANOVA) was run on the sequencing data after testing the data for normality. Treatments were compared using Tukey's test. Differences were considered significant at $p<0.05$. In addition, the statistical analysis of the Chao and Shannon index and quality of potato were compared between treatments by pairwise $t$-tests using SPSS (v.20.2).

\section{Conclusion}

In conclusion, the diversity of soil microbial community compositions was significantly different in treated soil relative to the untreated control. While the bacterial community diversity on the skin of potato was not altered, tuber quality improved, nonetheless. The results indicated that the variation of soil microbial community by Bacillus subtilis strain Bv17 treatment was able to decrease diseases of potato and improve the quality and quantity of yield, and thus, it may shed light on the regulatory roles of Bacillus subtilis strain Bv17 in the production of crops.

Supplementary Materials: The following are available online at https:/ /www.mdpi.com/article/ 10.3390/ijms222112065/s1; Figure S1: Venn diagram of bacterial OTUs shows the similarity and differences between the treated and control group; Figure S2: Venn diagram of fungal OTUs shows the similarity and differences between the treated and control group; Table S1: Statistics of sequencing data; Table S2: List of samples; Table S3: Statistics of $16 \mathrm{~S}$ sequencing data; Table S4: Statistics of ITS sequencing data; Table S5: 16S sequencing statistics of tags spliced by reads; Table S6: ITS sequencing statistics of tags spliced by reads; Table S7: Specific OTUs in soil sample of DT indicated bacteria in genus; Table S8: Specific OTUs in soil sample of DT indicated fungi in genus; Table S9: The species richness and diversity index of soil bacteria; Table S10: Statistics of $16 \mathrm{~S}$ sequencing data from potato samples; Table S11: The species richness and diversity index of potato bacteria; Table S12: Specific OTUs in potato sample of DT indicated bacteria in genus.

Author Contributions: J.-Y.C. and X.-F.D. conceived this research, J.-Y.C., J.S., Z.-Q.K. and R.L. designed and directed the study, D.-D.Z. assisted with specific experiments. J.-Y.C. and R.L. prepared the manuscript. J.S. and Z.-Q.K. contributed equally. All authors have read and agreed to the published version of the manuscript.

Funding: This work was supported by the National Key Research and Development Program of China (2018YFE0112500), the National Natural Science Foundation of China (31972228, 31970142), the Elite Youth Program CAAS to J.-Y.C., the Agricultural Science and Technology Innovation Program grant to X.-F.D.

Conflicts of Interest: The authors declare no conflict of interest.

\section{References}

1. Zarraonaindia, I.; Owens, S.M.; Weisenhorn, P.; West, K.; Marcell, J.H.; Lax, S.; Bokulich, N.A.; Mills, D.A.; Martin, G.; Taghavi, S.; et al. The soil microbiome influences grapevine-associated microbiota. mBio 2015, 6, e2527-14.

2. Trivedi, P.; Leach, J.E.; Tringe, S.G.; Sa, T.; Singh, B.K. Plant-microbiome interactions: From community assembly to plant health. Nat. Rev. Microbiol. 2020, 18, 607-621. [CrossRef]

3. Niu, B.; Paulson, J.N.; Zheng, X.; Kolter, R. Simplified and representative bacterial community of maize roots. Proc. Natl. Acad. Sci. USA 2017, 114, 2450-2459. [CrossRef]

4. Leach, J.E.; Triplett, L.R.; Argueso, C.T.; Trivedi, P. Communication in the Phytobiome. Cell 2017, 169, 587-596.

5. Mendes, R.; Garbeva, P.; Raaijmakers, J.M. The rhizosphere microbiome: Significance of plant beneficial, plant pathogenic, and human pathogenic microorganisms. FEMS Microbiol. Rev. 2013, 37, 634-663. [CrossRef] 
6. Cole, B.J.; Feltcher, M.E.; Waters, R.J.; Wetmore, K.M.; Mucyn, T.S.; Ryan, E.J.; Wang, G.; Hasan, S.U.; McDonald, M.; Yoshikuni, Y.; et al. Genome-wide identification of bacterial plant colonization genes. PLoS Biol. 2017, 15, e2002860. [CrossRef] [PubMed]

7. Levy, A.; Gonzalez, I.S.; Mittelviefhaus, M.; Clingenpeel, S.; Paredes, S.H.; Miao, J.; Wang, K.; Devescovi, G.; Stillman, K.; Monteiro, F.; et al. Genomic features of bacterial adaptation to plants. Nat. Genet. 2018, 50, 138-150. [CrossRef] [PubMed]

8. Pieterse, C.M.; Zamioudis, C.; Berendsen, R.L.; Weller, D.M.; Van Wees, S.C.M.; Bakker, P.A.H.M. Induced systemic resistance by beneficial microbes. Annu. Rev. Phytopathol. 2014, 52, 347-375. [PubMed]

9. Gouda, S.; Kerry, R.G.; Das, G.; Paramithiotis, S.; Shin, H.S.; Patra, J.K. Revitalization of plant growth promoting rhizobacteria for sustainable development in agriculture. Microbiol. Res. 2018, 206, 131-140. [CrossRef]

10. Xu, X.M.; Jeffries, P.; Pautasso, M.; Jeger, M.J. Combined use of biocontrol agents to manage plant diseases in theory and practice. Phytopathology 2011, 101, 1024-1031.

11. Mazzola, M. Assessment and Management of Soil Microbial Community Structure for Disease Suppression. Annu. Rev. Phytopathol. 2004, 42, 35-59. [CrossRef] [PubMed]

12. Santhanam, R.; Luu, V.T.; Weinhold, A.; Goldberg, J.; Oh, Y.; Baldwin, I.T. Native root-associated bacteria rescue a plant from a sudden-wilt disease that emerged during continuous cropping. Proc. Natl. Acad. Sci. USA 2015, 112, 5013-5020.

13. Van Bruggen, A.H.C.; Semenov, A.M.; van Diepeningen, A.D.; de Vos, O.J.; Blok, W.J. Relation between Soil Health, Wave-like Fluctuations in Microbial Populations, and Soil-borne Plant Disease Management. Eur. J. Plant Pathol. 2006, 115, 105-122.

14. Zhang, Y.; Xu, J.; Riera, N.; Jin, T.; Li, J.; Wang, N. Huanglongbing impairs the rhizosphere-to-rhizoplane enrichment process of the citrus root-associated microbiome. Microbiome 2017, 5, 97. [CrossRef] [PubMed]

15. Larousse, M.; Rancurel, C.; Syska, C.; Palero, F.; Etienne, C.; Industri, B.; Nesme, X.; Bardin, M.; Galiana, E. Tomato root microbiota and Phytophthora parasitica-associated disease. Microbiome 2017, 5, 56.

16. Shi, W.; Li, M.; Wei, G.; Tian, R.; Li, C.; Wang, B.; Lin, R.; Shi, C.; Chi, X.; Zhou, B.; et al. The occurrence of potato common scab correlates with the community composition and function of the geocaulosphere soil microbiome. Microbiome 2019, 7, 14.

17. Liu, K.; Mcinroy, J.A.; Hu, C.; Kloepper, J.W. Mixtures of Plant-Growth-Promoting Rhizobacteria Enhance Biological Control of Multiple Plant Diseases and Plant-Growth Promotion in the Presence of Pathogens. Plant Dis. 2018, 102, 67-72. [CrossRef]

18. Lugtenberg, B.; Kamilova, F. Plant-growth-promoting rhizobacteria. Annu. Rev. Microbiol. 2009, 63, 541-556. [CrossRef]

19. Ullah, A.; Nisar, M.; Ali, H.; Hazrat, A.; Hayat, K.; Keerio, A.A.; Ihsan, M.; Laiq, M.; Ullah, S.; Fahad, S.; et al. Drought tolerance improvement in plants: An endophytic bacterial approach. Appl. Microbiol. Biotechnol. 2019, 103, 7385-7397. [CrossRef] [PubMed]

20. Bamisile, B.S.; Dash, C.K.; Akutse, K.S.; Keppanan, R.; Afolabi, O.G.; Hussain, M.; Qasim, M.; Wang, L. Prospects of endophytic fungal entomopathogens as biocontrol and plant growth promoting agents: An insight on how artificial inoculation methods affect endophytic colonization of host plants. Microbiol. Res. 2018, 217, 34-50. [CrossRef]

21. Fira, D.; Dimkic, I.; Beric, T.; Lozo, J.; Stankovic, S. Biological control of plant pathogens by Bacillus species. J. Biotechnol. 2018, 285, 44-55. [CrossRef] [PubMed]

22. Cavalcanti, V.P.; Araujo, N.; Schwanestrada, K.; Pasqual, M.; Doria, J. Athelia (Sclerotium) rolfsii in Allium sativum: Potential biocontrol agents and their effects on plant metabolites. An. Acad. Bras. Cienc. 2018, 90, 3949-3962. [CrossRef] [PubMed]

23. Huang, S.; Pang, F. Biocontrol Agents for Controlling Wheat Rust. Methods Mol. Biol. 2017, 1659, $277-288$.

24. Amaresan, N.; Jayakumar, V.; Kumar, K.; Thajuddin, N. Biocontrol and plant growth-promoting ability of plant-associated bacteria from tomato (Lycopersicum esculentum) under field condition. Microb. Pathog. 2019, 136, 103713.

25. Iwama, K. Physiology of the Potato: New Insights into Root System and Repercussions for Crop Management. Potato Res. 2008, 51, 333-353.

26. Joshi, M.; Fogelman, E.; Belausov, E.; Ginzberg, I. Potato root system development and factors that determine its architecture. J. Plant Physiol. 2016, 205, 113-123. [CrossRef]

27. Weinert, N.; Piceno, Y.; Ding, G.C.; Meincke, R.; Heuer, H.; Berg, G.; Schloter, M.; Andersen, G.; Smalla, K. PhyloChip hybridization uncovered an enormous bacterial diversity in the rhizosphere of different potato cultivars: Many common and few cultivar-dependent taxa. FEMS Microbiol. Ecol. 2011, 75, 497-506. [PubMed]

28. Pfeiffer, S.; Mitter, B.; Oswald, A.; Hai, B.S.; Schloter, M.; Declerck, S.; Sessitsch, A. Rhizosphere microbiomes of potato cultivated in the High Andes show stable and dynamic core microbiomes with different responses to plant development. FEMS Microbiol. Ecol. 2017, 93, 242. [CrossRef]

29. Ji, X.B.; Wang, D.; Lu, Z.; Li, R.; Song, J.; Zhang, D.D.; Chen, J.Y.; Dai, X.F.; Lin, K.J. Inhibitory efficacy of BvR001 on Verticillium dahliae. Plant Prot. 2021, 47, 40-47.

30. Van Dingenen, J.; Hanzalova, K.; Abd Allah Salem, M.; Abel, C.; Seibert, T.; Giavalisco, P.; Wahl, V. Limited nitrogen availability has cultivar-dependent effects on potato tuber yield and tuber quality traits. Food Chem. 2019, 288, 170-177. [CrossRef]

31. Kong, H.G.; Kim, N.H.; Lee, S.Y.; Lee, S.W. Impact of a Recombinant Biocontrol Bacterium, Pseudomonas fluorescens pc78, on Microbial Community in Tomato Rhizosphere. Plant Pathol. J. 2016, 32, 136-144. [CrossRef] [PubMed]

32. He, A.; Sun, J.; Wang, X.; Zou, L.; Fu, B.; Chen, J. Reprogrammed endophytic microbial community in maize stalk induced by Trichoderma asperellum biocontrol agent against Fusarium diseases and mycotoxin accumulation. Fungal Biol. 2019, 123, 448-455. [CrossRef] [PubMed]

33. Gobbi, A.; Kyrkou, I.; Filippi, E.; Jensen, L.E.; Hansen, L.H. Seasonal epiphytic microbial dynamics on grapevine leaves under biocontrol and copper fungicide treatments. Sci. Rep. 2020, 10, 681. [CrossRef] [PubMed] 
34. Berg, G.; Koberl, M.; Rybakova, D.; Muller, H.; Grosch, R.; Smalla, K. Plant microbial diversity is suggested as the key to future biocontrol and health trends. FEMS Microbiol. Ecol. 2017, 93, fix050. [CrossRef]

35. Xie, Y.; Bu, H.; Feng, Q.; Wassie, M.; Amee, M.; Jiang, Y.; Bi, Y.; Hu, L.; Chen, L. Identification of Cd-resistant microorganisms from heavy metal-contaminated soil and its potential in promoting the growth and Cd accumulation of bermudagrass. Environ. Res. 2021, 200, 111730. [PubMed]

36. Leoni, C.; Piancone, E.; Sasanelli, N.; Bruno, G.L.; Manzari, C.; Pesole, G.; Ceci, L.R.; Velpicella, M. Plant Health and Rhizosphere Microbiome: Effects of the Bionematicide Aphanocladium album in Tomato Plants Infested by Meloidogyne javanica. Microorganisms 2020, 8, 1922. [CrossRef] [PubMed]

37. Berendsen, R.L.; Pieterse, C.M.J.; Bakker, P.A.H.M. The rhizosphere microbiome and plant health. Trends Plant Sci. 2012, 17, 478-486. [CrossRef]

38. Zhao, Y.; Fu, W.; Hu, C.; Chen, G.; Xiao, Z.; Chen, Y.; Wang, Z.; Cheng, H. Variation of rhizosphere microbial community in continuous mono-maize seed production. Sci. Rep. 2021, 11, 1544. [CrossRef]

39. Jiang, L.; Jeong, J.C.; Lee, J.S.; Park, J.M.; Yang, J.W.; Lee, M.H.; Choi, S.H.; Kim, C.Y.; Kim, D.H.; Kim, S.W.; et al. Potential of Pantoea dispersa as an effective biocontrol agent for black rot in sweet potato. Sci. Rep. 2019, 9, 16354. [CrossRef]

40. Raoul, D.E.Y.; Cigna, J.; Quetu Laurent, A.; Caron, A.; Munier, E.; Cirou, A.B.; Helias, A.; Faure, D. Biocontrol of the Potato Blackleg and Soft Rot Diseases Caused by Dickeya dianthicola. Appl. Environ. Microbiol. 2016, 82, 268-278.

41. Sarwar, A.; Latif, Z.; Zhang, S.; Hao, J.; Bechthold, A. A Potential Biocontrol Agent Streptomyces violaceusniger AC12AB for Managing Potato Common Scab. Front. Microbiol. 2019, 10, 202. [CrossRef]

42. Elazouni, I.; Abdel Aziz, S.; Rabea, A. Microbial efficacy as biological agents for potato enrichment as well as bio-controls against wilt disease caused by Ralstonia solanacearum. World J. Microbiol. Biotechnol. 2019, 35, 30. [PubMed]

43. Gupta, R.; Singh, A.; Srivastava, M.; Shanker, K.; Pandey, R. Plant-microbe interactions endorse growth by uplifting microbial community structure of Bacopa monnieri rhizosphere under nematode stress. Microbiol. Res. 2019, 218, 87-96. [CrossRef]

44. Baffoni, L.; Gaggia, F.; Dalanaj, N.; Prodi, A.; Nipoti, P.; Pisi, A.; Biavati, B.; Gioia, D. Microbial inoculants for the biocontrol of Fusarium spp. in durum wheat. BMC Microbiol. 2015, 15, 242.

45. Fadrosh, D.W.; Ma, B.; Gajer, P.; Sengamalay, N.; Ott, S.; Brotman, R.M.; Ravel, J. An improved dual-indexing approach for multiplexed 16S rRNA gene sequencing on the Illumina MiSeq platform. Microbiome 2014, 2, 6. [CrossRef]

46. Magoc, T.; Salzberg, S.L. FLASH: Fast length adjustment of short reads to improve genome assemblies. Bioinformatics 2011, 27, 2957-2963. [CrossRef]

47. Edgar, R.C. UPARSE: Highly accurate OTU sequences from microbial amplicon reads. Nat. Methods 2013, 10, 996-998. [CrossRef] [PubMed]

48. Schloss, P.D.; Westcott, S.L.; Ryabin, T.; Hall, J.R.; Hartmann, M.; Hollister, E.B.; Lesniewski, R.A.; Oakley, B.B.; Parks, D.H.; Robinson, C.J.; et al. Introducing mothur: Open-Source, Platform-Independent, Community-Supported Software for Describing and Comparing Microbial Communities. Appl. Environ. Microb. 2009, 75, 7537-7541. [CrossRef]

49. Tian, S.Y.; Zhang, Y.L.; He, T.J.; Niu, L.L.; Wang, Q.; Wu, Z.H.; Xiao, L.L.; Tian, S.J. Effects of potato-maize intercropping on potato growth, yield and carbohydrate. J. South. Agric. 2021, 52, 1198-1205. 